\title{
La política focalizada en el programa de vacunación contra el Vi- rus del Papiloma Humano en México: aspectos éticos
}

Kirvis Janneth Torres-Poveda ${ }^{1}$, Silvia Magali Cuadra-Hernández ${ }^{2}$, Julieta Ivone Castro-Romero ${ }^{1}$, Vicente Madrid-Marina $^{1}$

Resumen: Se plantea un análisis ético de aspectos relacionados con la introducción de nuevas vacunas contra el Virus del Papiloma Humano en México, cuya política de distribución atiende a la equidad más que a la igualdad y tiene como ejes fundamentales abarcar a las personas más vulnerables y al costo más bajo posible, es decir, atiende a los principios de justicia distributiva. El esquema inicial de vacunación contra este virus en México se ha focalizado a la población femenina más marginada, la cual se concentra en las mujeres indígenas. Las estrategias de distribución de nuevas vacunas deberán tomar en consideración las características específicas en que se desenvuelven estos grupos y analizar las implicaciones éticas que tales medidas conllevan. Sin ello, una política de salud pública podría aumentar las desigualdades en materia de salud.

Palabras clave: política de vacunación México, vacuna VPH, cuestionamientos éticos, inequidades y desigualdades en salud, justicia distributiva, política focalizada

\section{The policy focused on the HPV vaccination program against Human Papilloma Virus in Mexi- co: ethics aspects}

\begin{abstract}
This article raises an ethical analysis of issues related to the introduction of new vaccines against Human Papillomavirus in Mexico, whose distribution policy attend to equity more than equality, and has as fundamental axes to cover the most vulnerable people and the lowest cost possible, i.e. serving the principles of distributive justice. The initial scheme of vaccination against this virus in Mexico has been focused on the most marginalized female population, which focuses on indigenous women. Distribution strategies for new vaccines must take into account the specific characteristics that develop these groups and analyze the ethical implications that such measures will involve; without this public health policy could increase health inequalities.
\end{abstract}

Key words: Mexico's vaccination policy, HPV vaccine, ethical issues, inequities and inequalities in health, distributive justice, focalized policy

\section{Na política centrada no programa de vacinação contra o HPV no México: Suas dilema ético}

Resumo: O propósito foi fazer uma análise ética das questóes relacionadas com a introdução de novas vacinas contra o Papilomavírus Humano, no México, cuja política de distribuiçáo serve apenas para a equidade mais do que para a igualdade e tem como eixos fundamentais abrangir os mais vulneráveis e os menores custos possíveis, isto é, servindo aos princípios de justiça distributiva. O regime inicial da vacinação contra esse vírus no México tem sido focado na população feminina mais marginalizada, que incide sobre as mulheres indígenas. As estratégias de distribuiçẫo de novas vacinas devem tomar em consideração as características específicas que desses grupos e analisar as implicaçôes éticas que tais medidas implicam, sem isso a política de saúde pública poderia aumentar as desigualdades na saúde.

Palavras-chave: política de vacinaçáo México, Vacina contra o HPV, questões éticas, as iniqüidades e desigualdades em saúde, justiça distributiva, políticas orientadas.

\footnotetext{
${ }^{1}$ Dirección de Infecciones Crónicas y Cáncer, Centro de Investigaciones sobre Enfermedades Infecciosas, Instituto Nacional de Salud Pública (INSP), Cuernavaca, Morelos, México

Correspondencia: vmarina@insp.mx

${ }^{2}$ Centro de Investigaciones sobre Sistemas de Salud, INSP, Cuernavaca, Morelos, México
} 
Aspectos éticos del programa de vacunación contra el Virus del Papiloma Humano en México. Torres-Poveda, Cuadra-Hernández, CastroRomero y Madrid-Marina

\section{Introducción}

En países industrializados, las tasas de incidencia y mortalidad por cáncer cervicouterino $(\mathrm{CaCu})$ han disminuido en los últimos 50 ańos como resultado de la implementación de la prueba del $\mathrm{Pa}$ panicolau(1). En México, aunque el aumento de la cobertura del programa nacional de este tamizaje se ha reflejado en la disminución de la mortalidad global por $\mathrm{CaCu}(2)$, la mortalidad y la incidencia alta de casos en etapas avanzadas reflejan un bajo impacto, especialmente porque $90 \%$ de estos casos podrían ser detectados a través de Papanicolaou.

En México se han documentado disparidades regionales que han obstaculizado la garantía del acceso universal a los servicios de prevención, detección y tratamiento, y la reducción de la carga de la enfermedad en mujeres de clases socioeconómicas más marginadas, como sucede en otros países $(1,3)$.

Actualmente se vive una etapa histórica con relación al control del $\mathrm{CaCu}$, dado que existe un nuevo paradigma para la prevención primaria $y$ secundaria de la enfermedad, a partir de la asociación encontrada entre la presencia de Virus del Papiloma Humano (VPH) y este padecimiento. Por ello, se vislumbra una tendencia ascendente en el uso de las vacunas contra el VPH y las perspectivas de control de la infección(4). Así, la vacunación contra el VPH atiende a una necesidad de salud pública y es la única intervención preventiva factible en el ámbito poblacional que limita la exposición a la infección persistente por $\operatorname{VPH}(4,5)$.

Hasta el momento se han desarrollado dos vacunas profilácticas. Una es la tetravalente ${ }^{3}$, producida en levaduras que contiene "virus-like particles" (VLPs o partículas similares al virus) de dos genotipos de alto riesgo, VPH 16 y 18, responsables de $65 \%$ de las neoplasias en cérvix en Latinoamérica, y dos de bajo riesgo, VPH 6 y 11, responsables de $90 \%$ de las verrugas genitales y de la papilomatosis respiratoria recurrente. La vacuna bivalente ${ }^{4}$, ${ }^{3}$ Producida y distribuida por los laboratorios Merck and Co, Inc ${ }^{\circ}$, con un costo al público aproximado de 120 dólares americanos por dosis.

${ }^{4}$ A cargo de Glaxo Smith Kline Biologicals ${ }^{\circledast}$, con un costo al público similar a la vacuna tetravalente. que contiene VLPs de VPH 16 y 18 , se produce en células de insectos con baculovirus como sistema de expresión. Con base en resultados obtenidos en la fase tres de ensayos clínicos, en los cuales se ha evaluado la eficacia e inmunogenicidad de las vacunas de VPH, la Food and Drug Administration (FDA) de Estados Unidos de América otorgó licencia para el uso de estas vacunas en mujeres entre los 9 y 26 años de $\operatorname{edad}(6,7)$.

En los ensayos clínicos, el mayor impacto se ha visto en mujeres sin previa exposición al virus, por tanto, la edad promedio de inicio de las relaciones sexuales en las diferentes poblaciones es un elemento importante en las recomendaciones de la edad de vacunación. Lo que aún es materia de controversia es la vacunación en mujeres mayores de 26 años de edad. La posibilidad de que la protección en mujeres entre 15 y 26 años no sea conferida por los anticuerpos inducidos por la vacuna sino por anticuerpos adquiridos como consecuencia de una infección previa, y la carencia de efecto terapéutico de la vacuna(8) no revela el beneficio de la vacunación de este grupo de mujeres $y$, en contraposición, favorece la inversión de los recursos de los programas de salud pública para la vacunación de adolescentes antes de su debut sexual(9). La oferta de la vacuna a mujeres mayores de 26 años de edad sólo se hace a través de la práctica clínica privada.

El propósito de este artículo es revisar los aspectos éticos implicados en los esquemas de distribución de la vacunas profilácticas para VPH recomendados por las casas farmacéuticas productoras, el esquema de distribución de la vacuna para VPH de un modelo alterno al mexicano en la región de las Américas y, para el caso de la política de vacunación vigente en México, los dilemas éticos relacionados con la justicia en la distribución de la vacuna.

Así, la toma de decisiones para la asignación de recursos, en el caso de la vacuna del VPH, implica un dilema ético: dada la eficacia demostrada de la vacuna ¿se justifica en México optar por una política de vacunación focalizada o se debe vacunar a toda la población femenina? 


\section{Métodos}

Se realizó una revisión de fuentes secundarias acerca de los esquemas de distribución recomendados por las casas farmacéuticas productoras para las vacunas profilácticas contra VPH, el esquema de distribución canadiense de la vacuna para VPH como un modelo en la región de las Américas y el esquema de distribución propuesto para la actual política de vacunación contra el VPH en México. Se realizó un análisis de las recomendaciones actuales en México, se enlistan los cuestionamientos éticos relacionados con la propuesta de distribución y se discuten desde el enfoque de la justicia distributiva.

\section{Resultados}

Esquema de distribución recomendado por casas farmacéuticas productoras

En México, la Comisión Federal para la Protección contra Riesgos Sanitarios otorgó, en junio de 2006 y octubre de 2007, los registros sanitarios para las vacunas tetravalente y bivalente, respectivamente(10). Las casas farmacéuticas productoras de las vacunas profilácticas para VPH recomiendan administrar la vacuna tetravalente en tres dosis por vía intramuscular con el esquema de cero, 2 y 6 meses; y la bivalente con el esquema cero, 1 y 6 meses. Sólo así garantizan alcanzar la eficacia de $100 \%$ en la prevención de lesiones relacionadas con VPH 16-18 en mujeres, antes del inicio de su vida sexual, o que estén libres de la infección y una seroconversión para ambos tipos de VPH, 16 y 18 , después de la tercera dosis (mes siete), según los estudios clínicos realizados $(11,12)$.

Sin embargo, al precio actual de la vacuna ningún gobierno de la región de las Américas puede agregarla en sus programas debido a que su costo es ocho veces superior al resto de vacunas incluidas. El costo de dichas vacunas obedece a que las farmacéuticas productoras necesitan técnicas y tecnologías "extremadamente nuevas" para su producción.

\section{Modelo canadiense}

Canadá representa un escenario de referencia para el tamizaje de $\mathrm{CaCu}$. Los 10 ministerios de sanidad provinciales y los tres territoriales mantienen programas de tamizaje gratuitos. Sin embargo, se han topado con un umbral de rendimientos decrecientes dados los límites relacionados con la cobertura, la aceptabilidad y la sensibilidad baja de la citología. De tal manera, han complementado el programa de tamizaje de $\mathrm{CaCu}$ con la vacunación contra el VPH. El National Advisory Committee on Immunization de Canadá ha identificado la inmunogenicidad de dos dosis de vacuna y actualmente desarrolla una intervención en tres provincias de Canadá - British Columbia, Quebec y Nova Scotia-, bajo el esquema de vacunación a los cero, 6 y 60 meses(13). La diferencia respecto de lo recomendado por las casas farmacéuticas es el planteamiento de un esquema extendido de una segunda dosis a los 6 meses y una tercera de refuerzo a los 60 meses.

Esta iniciativa es una de las 10 más relevantes que se implementa sobre la vacuna contra el VPH en Canadá. Los argumentos para este tipo de esquema son los siguientes: a) la inducción de anticuerpos por la vacuna es más alta que la producida por la exposición natural al virus; b) la respuesta inmune de las niñas de 9 a 11 años de edad es similar a la obtenida después de tres dosis en mujeres entre los 16 y 26 años; c) de acuerdo con estudios puente, nada indica que la vacuna sea menos efectiva si se administra bajo un esquema extendido; d) la administración de la tercera dosis será en el momento en el que se requiere la máxima protección, es decir, alrededor del inicio de las relaciones sexuales, y e) una dosis administrada a los cinco años de la primera vacunación genera una respuesta de anticuerpos más alta que la obtenida durante la vacunación primaria(14).

El modelo de distribución de Canadá contempla en la actualidad el financiamiento de la vacuna contra VPH sin costo alguno por parte de las agencias provinciales de salud, para todas las estudiantes del grado octavo; esta selección se realizó debido a los altos costos que representa la administración de la vacuna. Los programas de vacunación escolares han constituido una vía efectiva para llegar a las muchachas jóvenes y asegurar la administración de todas las dosis requeridas de 
Aspectos éticos del programa de vacunación contra el Virus del Papiloma Humano en México. Torres-Poveda, Cuadra-Hernández, CastroRomero y Madrid-Marina

vacuna. Las mujeres entre 9 y 26 años de edad pueden acudir a su médico y pagar por recibir la vacuna, aunque en Canadá algunos planes de seguro médico cubren su costo(14).

Adicionalmente, en este país se recomienda la vacunación de rescate concomitante que consiste en administrar las tres dosis en un año, según lo recomendado por los fabricantes de vacunas, en mujeres de 13 a 26 ańos en cohortes anuales; esta decisión depende de los recursos presupuestarios disponibles y del número de mujeres jóvenes que ya no van a la escuela. Esta es considerada una medida adicional para prevenir situaciones de desigualdad(14).

En Canadá se han planteado y discutido diversos dilemas éticos frente a la vacunación contra VPH. Uno de ellos relacionado con la "promiscuidad sexual"; sin embargo, estas consideraciones no han tenido efecto al compararlas con el alto nivel de aceptación que tuvo entre padres y educadores la vacunación contra la hepatitis B provocada por un virus que también puede ser transmitido sexualmente(14).

\section{Modelo mexicano}

Basadas en el principio de que la salud es un derecho universal que trasciende fronteras, las actuales políticas de inmunización en la región latinoamericana son insuficientes para los retos que se observan en el contexto de la salud pública. Adicionalmente, los actuales mecanismos de cooperación multilateral económica-financiera para vacunación son inoperantes y han sido rebasados por una problemática cada vez más compleja.

La 58a Asamblea Mundial de la Salud aprobó la visión y estrategia mundial de inmunización enfocada a que en 2015 se dé alta prioridad a la inmunización, de manera que más personas estén protegidas contra un número mayor de enfermedades, se mantenga la inmunización en un marco de pluralidad de los valores sociales, de cambios demográficos, económicos y de evolución de las enfermedades, y que se haga una utilización óptima de las vacunas para mejorar la salud(15).
México ha sido considerado como país modelo del continente, por tener el esquema de vacunación más amplio, dado que presenta coberturas de vacunación superiores al $98 \%$ en niños menores de cinco años de edad. La vacunación ha sido una actividad de salud pública intensamente supervisada y evaluada en diferentes contextos, tanto por el nivel federal como por la Organización Panamericana de la Salud. El modelo seguido tradicionalmente en el diseño de políticas de vacunación en México es el de la justicia como bienestar colectivo; tal como lo plantean Figueroa y Sánchez, "se busca corregir las consecuencias del libre mercado con un principio de justicia redistributiva, cambiando la concepción de la salud como problema privado a interpretarlo como un problema público a través de políticas de bienestar social que reconozcan al ser humano como sujeto de derecho y a la justicia para regular y expresar el bien colectivo"(16:90).

Sin embargo, México no es ajeno a la problemática que representa el alto costo y financiamiento de las nuevas vacunas y la remoción de las barreras regulatorias que obstaculizan su introducción. Para garantizar la sustentabilidad a largo plazo de nuevos esquemas de vacunación, como lo es el del VPH, el financiamiento de las vacunas debe provenir de fondos públicos, los cuales deberán ser asignados en el presupuesto por el Poder Legislativo, de tal forma que la asignación responda a criterios de política pública en salud y se aleje de modelos relacionados con el libre mercado o con el asistencialismo.

Sin embargo, en México no se cuenta con los medios necesarios, tanto en el marco legal como financiero, para garantizar que este financiamiento sea progresivo e irreversible. Para el caso del programa de prevención y control de $\mathrm{CaCu}$ en México, la asignación de recursos proviene de manera directa del Congreso de la Unión, quienes, si consideran pertinente, realizan recortes presupuestarios en algunos programas de inmunización existentes para darle prioridad a otros problemas urgentes; un ejemplo puede ser el relativo a los recursos necesarios para vacunación en la contingencia de influenza AH1N1 pandémica. 
Adicionalmente, se presenta un conflicto de intereses entre los distintos actores en los procesos de inmunización (gobierno, industria farmacéutica y distribuidores) sobre el precio adecuado de cada vacuna o sobre los requisitos que deben cubrirse previo a la certificación, producción y uso de una vacuna. La introducción de una nueva vacuna dentro del presupuesto asignado al Programa Nacional de Vacunación constituye un gran desafío. Con frecuencia, el sector privado se encuentra mejor posicionado que el Poder Legislativo para cabildear políticas relacionadas con las prácticas de inmunización.

El costo promedio de la vacuna en México, independientemente de la casa farmacéutica, es de 120 dólares por cada dosis; si se requieren tres dosis para la administración del esquema completo, el costo promedio asciende a 360 dólares americanos. Este costo de por sí ya es elevado para la población femenina mexicana en general e inaccesible para las mujeres que viven en áreas de marginación alta, las más proclives a padecer y morir por $\mathrm{CaCu}$ (3). Según el Consejo Nacional de Población, en México la marginación en las localidades con $40 \%$ o más de hablantes de lenguas indígenas es muy alta (85\%) y $77 \%$ de la población económicamente activa en las áreas marginadas recibe ingresos inferiores al salario mínimo $(17,18)$.

El escenario de vacunación universal, con una cobertura de $80 \%$ como meta para una intervención dirigida a adolescentes, no resulta plausible si se mantienen los precios ofertados por dosis de vacuna, lo que hace financieramente inviable su introducción y operación mientras no estén cercanos al umbral calculado de costo-efectividad en México de 17 dólares americanos por dosis(19).

Dicho precio es uno de los factores relevantes que deben considerarse antes de su introducción al sector público mexicano. Empero, una evaluación completa del costo del programa de vacunación contra VPH incluiría además los costos de la posible aplicación de una dosis de refuerzo a los cinco años tras completar el esquema básico, con el fin de mantener y prolongar la inmunidad de la vacuna(20), así como los costos de implemen- tación del programa y de la estrategia educativa dirigida a población y prestadores de servicios, lo que representa costos adicionales al presupuestar la vacuna.

Aun cuando se tenga una estimación de los costos en salud, se reconozcan los beneficios de incluir la vacuna en el programa nacional y ésta haya sido aprobada bajo el marco de un análisis de costoefectividad y costo-beneficio, existen barreras regulatorias que impiden que las autoridades encargadas de la asignación presupuestaria otorguen fondos adicionales necesarios para introducir el nuevo biológico en el programa nacional de inmunización.

Mientras que ciertos países de ingreso bajo se han visto beneficiados por los esquemas multilaterales de respaldo a las prácticas de inmunización, incluyendo los aspectos de financiamiento y logística, los países de ingreso medio, como México, son muchas veces excluidos de estos esquemas y no tienen posibilidad de encontrar otros fondos de ayuda. Al respecto, México ha ido incorporando decisiones de política pública para la introducción de vacunas en sus programas de inmunización, con base en un sustento científico y económico(21).

La recomendación actual de vacunación para VPH en México no sigue las recomendaciones de las casas farmacéuticas, sino el modelo de distribución canadiense de cero, 6 y 60 meses, pero en niñas entre 9 y 12 años, dirigido de manera prioritaria y focalizada a mujeres jóvenes de comunidades indígenas - con el objeto de reducir el número de casos sin tratamiento de $\mathrm{CaCu}$, en una población que tradicionalmente experimenta problemas de acceso a servicios de tamizaje y tratamiento- y a mujeres jóvenes en comunidades urbano-marginadas donde potencialmente se ubica la población con la mayor prevalencia y un riesgo alto de infección por $\mathrm{VPH}(22)$. El esquema de distribución adoptado en México incrementará la cobertura de prevención primaria en niñas a los nueve ańos de edad y, al diferir la tercera dosis 60 meses, se reducirán costos. De tal manera que los ahorros generados podrán fortalecer la detección secundaria de $\mathrm{CaCu}$. El plan sólo incluye 
Aspectos éticos del programa de vacunación contra el Virus del Papiloma Humano en México. Torres-Poveda, Cuadra-Hernández, CastroRomero y Madrid-Marina

a mujeres, ya que la efectividad de la vacuna en hombres no ha sido establecida de manera definitiva. Sin embargo, no se descarta por completo su potencial beneficio para los hombres, o el beneficio acumulado para las mujeres cuando también se vacune a los hombres.

\section{Aspectos éticos: apuntes para una discusión}

El grupo de expertos que definió las recomendaciones para la vacunación de VPH en México formuló el escenario de replicar el esquema extendido de vacunación contra VPH de cero, 6 y 60 meses de Canadá (de acuerdo con los resultados de inmunogenicidad ya publicados), pero de manera prioritaria y focalizada a nińas de 9 y 12 años de comunidades indígenas y comunidades urbanomarginadas más que un esquema universal, dado el costo que representaría para el sistema de salud la vacunación masiva, el presupuesto limitado disponible para este rubro y, particularmente, por las siguientes consideraciones:

Cobertura universal vs. focalizada: la estrategia generalmente adoptada para prevenir enfermedades transmisibles es la vacunación masiva sustentada en el principio de solidaridad, que asegura niveles de protección efectiva a la mayoría de la población. Una alternativa es la estrategia de vacunación selectiva que apunta a aquellos individuos con mayor riesgo de adquirir la enfermedad y/o a quienes, por factores constitucionales o adquiridos, tal patología generaría complicaciones graves (23).

En México existen disparidades sociales en el acceso al tamizaje y tratamiento del $\mathrm{CaCu}$, sobre todo en mujeres de estratos socioeconómicos bajos (mujeres indígenas y mujeres jóvenes de comunidades urbano-marginadas $(3,24)$ ), lo que confiere a esta población un riesgo mayor de que no se les detecte oportunamente una infección persistente por VPH oncogénico, el desarrollo de una lesión premaligna y posterior cáncer en cuello uterino. Las mujeres que viven en áreas marginadas y geográficamente inaccesibles suelen presentar factores de riesgo nutricionales que las hacen tener una respuesta inmune limitada frente a una infección persistente por $\operatorname{VPH}(3,25)$.
Si se tiene en cuenta que la alternativa más viable para la disminución de la morbilidad y mortalidad por $\mathrm{CaCu}$ es el control de la infección por el VPH, mediante la detección temprana de éste y la prevención de la infección con vacunas profilácticas, en el caso de que una mujer indígena se infectara con el VPH tiene menos probabilidad de que se le detecte la infección y reciba tratamiento oportuno, comparada con una mujer de área urbana, debido a que la ubicación de las poblaciones indígenas es predominantemente rural, donde los servicios de salud se encuentran inaccesibles y existe una falta de consideración de los aspectos culturales que inciden en el proceso salud-enfermedad-atención (conocimiento colectivo, estrategias comunitarias, prácticas ancestrales e insumos naturales), así como de la organización y prestación de los servicios de salud (discriminación institucional, deficiencias en la relación médico-paciente), que han dado lugar a una cobertura insuficiente de los servicios básicos proporcionados a esta población(26). De esta manera, ofrecer el esquema de vacunación a mujeres indígenas antes del inicio de su vida sexual representa una ventana de protección para ellas frente a infecciones futuras por los principales virus oncogénicos.

La decisión de optar por una política focalizada, en el programa de vacunación para VPH en México, lejos de ser interpretada como paternalista puede sustentarse en que la inclusión de la vacuna contra VPH no debe perpetuar las injusticias en salud, sino introducirse estratégicamente de tal manera que se beneficie a la población en mayor riesgo de infección por VPH. Por esta razón, es más viable, dentro de los principios de la justicia distributiva y la equidad en las políticas de salud, optar por políticas focalizadas, al buscar lo mejor para la minoría en riesgo.

Aunque, en el plano mundial, los programas exitosos para reducir la incidencia de $\mathrm{CaCu}$ se han basado en estrategias mejoradas de tamizaje, seguimiento y tratamiento, en el caso de México se invierten recursos públicos para financiar una política de vacunación focalizada contra VPH, dado que en la población más afectada por esta 
enfermedad en términos de mortalidad — mujer en área marginada y mujer indígena- se han demostrado inequidades en el acceso a los programas de tamizaje de $\mathrm{CaCu}(3,25)$. Uno de los grupos sociales que se encuentra rezagado respecto a la atención en salud sexual y reproductiva es la población indígena, sobre la cual se ha evidenciado el trato discriminatorio de los servicios de salud institucionales(27).

Igualdad vs. Equidad: tal como define Montt Momberg, "los principios de dignidad, justicia, equidad, solidaridad, participación y ética profesional son valores que deben guiar las políticas y organizaciones de salud para crear las condiciones necesarias para alcanzar la igualdad de oportunidades"(28:86). No obstante, la recomendación de vacunación para VPH en México no atiende a la igualdad; por el contrario, sigue el concepto aristotélico de justicia distributiva y la aplicación del criterio de equidad dentro del marco de la justicia social, el cual, según Rawls, "representa entregar el máximo beneficio a los menos aventajados, sin descuidar al resto de la sociedad" (29:68). La equidad representa un imperativo de carácter ético, asociado con principios de justicia social y derechos humanos, de tal manera que se traduce en la minimización de disparidades evitables en salud y sus determinantes entre grupos humanos con diferentes niveles de privilegio social.

Asimismo, bajo el concepto de equidad en salud, los organismos internacionales definen que "las necesidades de las personas son las que deben guiar la distribución de las oportunidades para el bienestar"(30). De tal forma, las recomendaciones actuales para la vacunación de VPH en México se sustentan en la noción de necesidad de las mujeres mexicanas que viven en áreas de alta marginación, con base en el concepto de equidad distributiva. Tal noción apunta hacia una distribución de recursos no de tipo igualitario, sino de asignación diferencial de acuerdo con los requerimientos particulares de esos grupos e individuos.

La base de la relación entre género y desarrollo de la salud es la visión de una sociedad más equitativa, con una distribución más justa de los recursos y beneficios de ese desarrollo y con una mayor participación de las mujeres, especialmente las de los sectores menos privilegiados(31).

Asignación de recursos: es esencial para el desarrollo de políticas de salud la forma de asignación de recursos. No sólo implica comparar costos, se requiere de la aplicación de los principios éticos de justicia, equidad, universalidad, igualdad de oportunidades, solidaridad y participación, para equilibrar los derechos universales y colectivos. El valor de la solidaridad debe tomarse en cuenta particularmente en las políticas de vacunación, en las que se exige una asignación más justa de los recursos(28).

Los costos son un factor limitante decisivo en el acceso a la vacuna contra VPH. El gobierno mexicano debe garantizar la sustentabilidad del programa de vacunación contra VPH, teniendo en cuenta que constituye una inversión, al permitir ahorrar en gastos médicos, años saludables perdidos y sufrimiento evitado. Una alternativa es la creación de fondos rotativos internacionales que posibiliten compras masivas y reduzcan costos(32).

En la actualidad, un escenario de cobertura universal de vacunación frente a VPH en México no es viable, dado los altos costos de la vacuna. Por lo que la opción más costo-efectiva, por el momento, es seguir una estrategia de vacunación selectiva en aquellos individuos en los cuales, si se adquiriera una infección persistente por $\mathrm{VPH}$ de alto riesgo, la posibilidad de detección y tratamiento oportuno antes de la progresión a lesión premaligna y maligna estuviera limitada.

Respeto a las tradiciones culturales de los pueblos: la cultura de cada grupo humano debe ser tomada en cuenta al planificar y ejecutar un programa de vacunación, por lo que, en la implementación de las recomendaciones de vacunación en México, se han tenido en cuenta los conceptos sociales sobre salud, los prejuicios y la representación antropológica del cuerpo, que pueden volverse condicionantes para la aceptabilidad de las vacunas, sobre todo en la población indígena, que es la más susceptible a desarrollar $\mathrm{CaCu}$ en México(33). El impacto de la vacuna en el comportamiento 
Aspectos éticos del programa de vacunación contra el Virus del Papiloma Humano en México. Torres-Poveda, Cuadra-Hernández, CastroRomero y Madrid-Marina

sexual de los adolescentes(34) y el grado de conocimientos acerca de $\mathrm{CaCu}$ y el VPH son temas de gran importancia respecto de la aceptación de la vacuna por parte de los entes gubernamentales, los padres de familia y el adolescente(35).

\section{Conclusiones}

La eliminación de los obstáculos que dificultan un acceso pleno a los avances médicos está en la mira de los programas de control de enfermedades (especialmente de las inmunoprevenibles) que, a su vez, constituyen parte de las iniciativas para superar la exclusión social, la pobreza y la mortalidad evitable.

La recomendación actual para la distribución de la vacuna de VPH en México sigue un esquema equitativo más que igualitario y tiene como ejes fundamentales abarcar a las personas más vulnerables y al costo más bajo posible, es decir, atiende a los principios de justicia distributiva.

Condiciones de vulnerabilidad como la etnicidad, nivel socioeconómico y el acceso limitado a los servicios de salud de la población indígena, además de la falta de recursos adquisitivos de la población femenina de áreas urbano marginadas, hace a estas poblaciones blancos para focalizar la asignación de recursos en la política de vacunación contra VPH en México. No obstante, la política de vacunación contra VPH y el programa de tamizaje del mismo no son excluyentes, son aditivos frente al reto que representa disminuir los índices de morbilidad y mortalidad por $\mathrm{CaCu}$ en México. Sólo así se contribuirá a dar una respuesta social organizada en beneficio de la salud de la población femenina más vulnerable.

Por ello, el reto es combinar una política universal de vacunación contra VPH con una política diferencial, capaz de satisfacer necesidades particulares de los grupos socialmente excluidos.

\section{Agradecimientos}

Este trabajo fue apoyado por la Beca Forgaty. Grant: 5 R25 TW006056-08, Programa de Posgrado en Bioética de Investigación, Universidad de Chile, Santiago de Chile.

\section{Referencias}

1. Organización Mundial de la Salud. Integración de la atención sanitaria para la salud sexual y reproductiva y las enfermedades crónicas. Control integral del cáncer cérvicouterino. Guía de prácticas esenciales 2007; 281. Disponible en http://www.rho.org/files/WHO_CC_control_ spanish_2007.pdf

2. Lazcano-Ponce E, Palacio-Mejía L, Allen-Leigh B, Yunes-Díaz E, Alonso P, Schiavon R, et al. Decreasing cervical cancer mortality in Mexico: Effect of Papanicolaou coverage, birthrate, and the importance of diagnostic validity of cytology. Cancer Epidemiology Biomarkers \& Prevention 2008;17(10): 2808-2817.

3. Palacio-Mejía L, Rangel-Gómez G, Hernández-Ávila M, Lazcano-Ponce M. Cervical cancer, a disease of poverty: Mortality differences between urban and rural areas in Mexico. Salud Pública de México 2003; 45(3): 315-325.

4. Madrid-Marina V, Torres-Poveda K, López-Toledo G, García-Carrancá A. Advantages and disadvantages of current prophylactic vaccines against HPV. Archives of Medical Research 2009; 40: 471-477.

5. Organización Mundial de la Salud. Human papillomavirus and HPV vaccines: Technical information for policy-makers and health professionals; 2007: 43. Disponible en http://whqlibdoc.who.int/ hq/2007/WHO_IVB_07.05_eng.pdf

6. Food and Drug Administration. Product approval information - licensing action [package insert]. Gardasil (quadrivalent human papillomavirus types 6,11,16,18). 2007. Available from: http:// www.fda.gov/cber/label/ HPVmer060806LB.pdf. 
7. Cervarix ${ }^{\oplus}$ U.S. regulatory update. Available from: http://www.gsk.com/media/ pressreleases/2009/2009_pressrelease_10038.htm.

8. Hildesheim A, Herrero R, Wacholder S, Rodríguez AC, Solomon D, Bratti MC, et al. Effect of human papillomavirus 16/18 L1 virus-like particle vaccine among young women with preexisting infection: A randomized trial. Journal of the American Medical Association 2007; 29(7) 8: 743-753.

9. Hildesheim A, Herrero R. Human papillomavirus vaccine should be given before sexual debut for maximum benefit. Journal of Infectious Diseases 2007; 196(10): 1431-1432.

10. Secretaría de Salud de México. Comisión federal para la protección contra riesgos sanitarios. Vacunas autorizadas en México; 2008. Disponible en http://www.salud.gob.mx/unidades/cofepris/inf/ vacunas/VacunasRegistradas.htm

11. Paavonen J, Jenkins D, Bosch FX, Naud P, Salmerón J, Wheeler CM, et al. Efficacy of a prophylactic adjuvanted bivalent L1 virus-like-particle vaccine against infection with human papillomavirus types 16 and 18 in young women: An interim analysis of a phase III double-blind, randomised controlled trial. The Lancet 2007; 369(9580): 2161-2170.

12. Pérez G, Lazcano-Ponce E, Hernández-Ávila M, García PJ, Muñoz N, Villa LL, et al. Safety, immunogenicity, and efficacy of quadrivalent human papillomavirus (types 6, 11, 16, 18) L1 virus-like-particle vaccine in Latin American women. International Journal of Cancer 2008; 122(6): 1311-1318.

13. Agence de la santé et des services sociaux de Montréal. Québec: HVP Vaccination. Questions and Answers for Practicioners. Available from: URL: http://www.santepub-mtl.qc.ca/Mi/vaccination/ pdf/VPHenglish2008.pdf

14. Steben M. El paradigma de Canadá para la vacunación VPH y el programa en Quebec. Newsletter on Human Papillomavirus. HPV Today 2008; 15: 6-7.

15. Organización Mundial de la Salud. Preparación de la introducción de las vacunas contra el virus del papiloma humano. Orientaciones normativas y programáticas para los paises; 2006: 24. Disponible en http://screening.iarc.fr/doc/hpvvaccine_es.pdf

16. Figueroa JG, Sánchez V. Algunas reflexiones sobre ética, derechos humanos y salud. En: Bronfman M, Castro R, (eds.) Salud, cambio social y politicas. Perspectivas desde América Latina. 2a ed. México DF: Edamex; 1999: 81-95.

17. Consejo Nacional de Población. Índices de marginación México 2000. Disponible en http://www-. conapo.gob.mx/publicaciones/indice2000.htm

18. Ramos R. Necesidades esenciales en México: situación actual y perspectivas en el año 2000. En: Boltvinik J, eds. Geografía de la marginación. 5a ed. México DF: Siglo Veintiuno Editores; 1999: 68.

19. Gutiérrez-Delgado C, Báez-Mendoza C, González-Pier E, Prieto-de la Rosa A, Witlen R. Costoefectividad generalizado de intervenciones preventivas contra el cáncer cervical en mujeres mexicanas: resultados de un modelo markoviano con la perspectiva del sector público. Salud Pública de México 2008; 50(2): 107-118.

20. Olsson SE, Villa LL, Costa RL, Petta CA, Andrade RP, Malm C, et al. Induction of immune memory following administration of a prophylactic quadrivalent human papillomavirus (HPV) types 6/11/16/18 L1 virus-like particle (VLP) vaccine. Vaccine 2007; 25(26): 4931-4939.

21. Lozano R, Soliz P, Gakidou E, Abbott-Klafter J, Feehan DM, Vidal C, et al. Benchmarking of performance of Mexican states with effective coverage. The Lancet 2006; 368(9548): 1729-1741.

22. Lazcano-Ponce E, Salmerón-Castro J, García-Carrancá A, Aranda-Flores C, Madrid-Marina V, Gómez-Altamirano C, et al. Recomendaciones para política de vacunación de VPH en México. Salud Pública de México 2009; 51(4): 336-341.

23. Malone K, Hinman A. Vaccination mandates: The Public Health Imperative and Individual Rights, In: Goodman RA, Rothstein MA, Hoffman RE, (eds.) Law in Public Health Practice. New York: Oxford University Press; 2003: 262-284. 
Aspectos éticos del programa de vacunación contra el Virus del Papiloma Humano en México. Torres-Poveda, Cuadra-Hernández, CastroRomero y Madrid-Marina

24. Montenegro RA, Stephens C. Indigenous health in Latin America and the Caribbean. The Lancet 2006; 367(9525): 1859-1869.

25. Givaudan M, Pick S, Poortinga YH, Fuertes C, Gold L. A cervical cancer prevention programme in rural Mexico: Addressing women and their context. Journal of Community \& Applied Social Psychology 2005; 15(5): 338-352.

26. Figuerasa P, Musgroveb G, Carrinc D. Retos para los sistemas sanitarios de Latinoamérica: ¿qué puede aprenderse de la experiencia europea? Gaceta Sanitaria 2002; 16(1): 5-17.

27. Secretaría de Salud. Interculturalidad en Salud. Experiencias y aportes para el fortalecimiento de los servicios de salud; 2008: 176.

28. Montt-Momberg J. Políticas de salud: ética en la asignación de recursos, En: Ética y Salud; 1997: 81.

29. Rawls J. Teoría de la justicia. 2a ed. México DF: Fondo de Cultura Económica; 1995: 31.

30. United Nations. The International Forum for Social Development Social Justice in an Open World. The Role of the United Nations; 2006: 1-20. Available from: URL: http://www.un.org/esa/socdev/ documents/ifsd/SocialJustice.pdf.

31. OPS. Equidad de género en salud. Programa Mujer, Salud y Desarrollo; 8: 1. Disponible en http:// www.paho.org/spanish/hdp/hdw/GEHPaperSpanish.pdf

32. Mandelblatt JS, Lawrence WF, Gaffikin L, Limpahayom KK, Lumbiganon P, et al. Costs and benefits of different strategies to screen for cervical cancer in less-developed countries. Journal of the National Cancer Institute 2002; 94(19): 1469-1483.

33. Torres-Poveda KJ, Arredondo A, Duarte MB, Madrid-Marina V. La mujer indígena, vulnerable a cáncer cervicouterino: perspectiva desde modelos conceptuales de salud pública. Revista Salud en Tabasco 2008; 14(3): 807-815.

34. Devereaux Ch, Gera A, Shah M, Sharma A, Powell JE, Wilson S. Public knowledge and attitudes towards Human Papilloma Virus (HPV) vaccination. BMC Public Health 2008; 8: 368.

35. Brotherton JM, Gold MS, Kemp AS, Mclntyre PB, Burgess MA, Campbell-Lloyd S; New South Wales Health HPV Adverse Events Panel. Anaphylaxis following quadrivalent human papillomavirus vaccination. Canadian Medical Association Journal 2008; 179(6): 525-533.

Recibido: 21 de enero de 2010

Aceptado: 13 de marzo de 2010 